\title{
Tunneling Effect from a Non-static Black Hole with the Internal Global Monopole
}

\author{
Ren Jun
}

Received: 25 February 2008 / Accepted: 2 May 2008 / Published online: 31 May 2008

(C) The Author(s) 2008. This article is published with open access at Springerlink.com

\begin{abstract}
In this paper, we extend Parikh' work to the non-stationary black hole, a non-static black hole with the internal global monopole. We view Hawking radiation as a tunneling process across the event horizon and calculate the tunneling probability. We find that the result is different from Parikh's work because $\frac{d r_{H}}{d v}$ is the function of Bondi mass $m(v)$.
\end{abstract}

Keywords Tunneling effect · Information conservation $\cdot$ Non-stationary black hole

\section{Introduction}

Steven Hawking' discovery that basic principles of quantum field theory could lead to the emission of thermal radiation from a classical black hole [1] in the 1970s. At the same time, it sets up a disturbing and difficult problem: information loss paradox. Moreover, when Hawking first proved the existence of black hole radiation, he described it as tunneling triggered by vacuum fluctuations near the horizon. But, actual derivation of Hawking radiation did not proceed in this way at all. There were two difficulties to overcome. The first was that in order to do a tunneling computation, one needed to find a coordinate system which was well-behaved at the event horizon. The second was that there did not seem to be any barrier.

Recently, a method to describe Hawking radiation as a tunneling process where a particle moves in dynamic geometry was developed by Kraus and Wilczek [2] and elaborated upon by Parikh and Wilczek [3-5]. In their method, they take the self-gravitation into account and it is the tunneling particle itself that defines the barrier [5]. And they also give a leading correction to the emission rate arising from loss of mass of the black hole corresponding to the energy carried by the radiated quantum. Following this method, the concerned particles were published [6, 7]. Especially, Zhang and Zhao made much progress. They extended Parikh's method from static black holes to the non-spherical symmetric stationary black holes and radiation of charged particle and massive particle [8-11]. In all these investigations, however, all the black holes are static. In this paper, we will extend the investigation

R. Jun $(\bowtie)$

School of Science, Hebei University of Technology, Tianjin 300130, China

e-mail: thebest2@163.com 
to a non-stationary black hole- the non-static black hole with the internal global monopole. There are two crucial points. First, the non-stationary black hole radiates energy continuously and there is a problem how to use the condition of the energy conservation. In quantum mechanics, particle tunneling a barrier is a instantaneous process, so, the metric in the coordinates should satisfy Landau' coordinate clock synchronization condition. Although not all the non-stationary black holes satisfy Landau' condition, the black hole which is studied in this paper does satisfy it [14]. Consequently, we could fix a certain Bondi time $v^{\prime}$ and at this time $v^{\prime}$ the Bondi energy is conserved. Second, although the components of the metric in the advanced Eddington coordinate system are not singular, the event horizon and the time like limit surface do not locate at the same place, which causes difficulty in the calculation of the emission rate. We have to introduce a new coordinate, $R=r-r_{H}(v)$. In the new coordinate system, the event horizon and the time like limit surface do locate at the same place. After calculation, the result is different from Parikh's work because $\dot{r}_{H}$ is the function of $m(v)$. We emphasize that although the calculation has been employed at the moment $v^{\prime}$, $v^{\prime}$ is chosen arbitrarily. So, our results reveal the dynamical change of the non-stationary black hole. Throughout the paper, the units $G=c=\hbar=1$ are used.

\section{New Coordinates}

The metric of the non-static black hole with the internal global monopole can be written as [12]

$$
d s^{2}=-\left[1-8 \pi \eta^{2}-\frac{2 m(v)}{r}\right] d v^{2}+2 d v d r+r^{2}\left(d \theta^{2}+\sin ^{2} \theta d \varphi^{2}\right),
$$

where $m(v)$ is Bondi mass and $\eta$ is an internal global monopole which is unconcerned with time $v$. From $g_{00}=0$, we get, $r=2 m(v) /\left(1-8 \pi \eta^{2}\right)$, which is the time like limit surface, not the event horizon. If we define a new radical coordinate [15] $R=r-r_{H}(v)$, the line element (1) can be rewritten as

$$
\begin{aligned}
d s^{2} & =-\left[1-8 \pi \eta^{2}-\frac{2 m(v)}{r}-2 \dot{r}_{H}\right] d v^{2}+2 d v d R+r^{2}\left(d \theta^{2}+\sin ^{2} \theta d \varphi^{2}\right) \\
& =\tilde{g}_{00} d v^{2}+2 \tilde{g}_{01} d v d R+\tilde{g}_{22} d \theta^{2}+\tilde{g}_{33} d \varphi^{2}
\end{aligned}
$$

The event horizon is $[12,13]$

$$
r_{H}=\frac{2 m(v)}{1-8 \pi \eta^{2}-2 \dot{r}_{H}}
$$

where $\dot{r}_{H} \equiv \frac{d r_{H}}{d v}$. In the new coordinates, from $\tilde{g}_{00}=0$ we can obtain the equation of event horizon, $r_{H}=\frac{2 m(v)}{1-8 \pi \eta^{2}-2 \dot{r}_{H}}$. So, the event horizon and the time like limit surface locate at the same place in the new coordinates. The reason why we must adopt the new coordinates will be explained in Sect. 3. Because particle tunneling a barrier is a instantaneous process, we will verify that the metric in new coordinate system satisfy Landau' coordinate clock synchronization condition as follows.

According to Landau' coordinate clock synchronization theory, in a space-time decomposed in $3+1$, the difference of coordinate times of two events taking place simultaneously in different place is $[16,17]$

$$
\Delta T=-\int \frac{g_{0 i}}{g_{00}} d x^{i}
$$


where $i=1,2,3$. If the simultaneity of coordinate clocks can be transmitted from one place to another and have nothing to do with the integration path, components of the metric should satisfy

$$
\begin{gathered}
\frac{\partial}{\partial x^{j}}\left(\frac{g_{0 i}}{g_{00}}\right)=\frac{\partial}{\partial x^{i}}\left(\frac{g_{0 j}}{g_{00}}\right), \\
i, j=1,2,3 .
\end{gathered}
$$

In this case, $x^{1}=r, x^{2}=\theta, x^{3}=\varphi$, so we can obtain

$$
\frac{\partial}{\partial x^{2}}\left(\frac{g_{01}}{g_{00}}\right)=\frac{\partial}{\partial x^{1}}\left(\frac{g_{02}}{g_{00}}\right)=\frac{\partial}{\partial x^{3}}\left(\frac{g_{01}}{g_{00}}\right)=\frac{\partial}{\partial x^{1}}\left(\frac{g_{03}}{g_{00}}\right)=0 .
$$

That is to say, we can define coordinate clock synchronization although the space-time is non-stationary. This feature of the coordinates is very important for us to discuss the tunneling process. The area of the event horizon $A_{H}$, the entropy [12] of the black hole are given by

$$
\begin{aligned}
A_{H} & =\int_{r_{H}} \sqrt{-g} d \theta d \varphi=\frac{16 \pi m^{2}(v)}{\left(1-8 \pi \eta^{2}-2 \dot{r}_{H}\right)^{2}}, \\
S & =\frac{1}{4} A_{H}=\frac{4 \pi m^{2}(v)}{\left(1-8 \pi \eta^{2}-2 \dot{r}_{H}\right)^{2}} .
\end{aligned}
$$

To describe across-horizon phenomena, it is necessary to choose coordinates which are not singular at the horizon; fortunately the line element (2) is no singular at the horizon $r_{H}=\frac{2 m(v)}{1-2 \dot{r}_{H}}$. The radial null geodesics are given by

$$
\dot{R} \equiv \frac{d R}{d v}=\frac{1}{2}\left[1-8 \pi \eta^{2}-\frac{2 m(v)}{r}-2 \dot{r}_{H}\right]
$$

corresponding to outgoing geodesics, where $v$ increases towards the future. These equations are modified when the particle's self-gravitation is taken into account. We can consider the particle as a shell of energy. We fix the total mass (Bondi mass) and allow the hole mass to fluctuate. When the shell of energy $\omega$ travels on the geodesics, we should replace $m\left(v^{\prime}\right)$ with $m\left(v^{\prime}\right)-\omega$ in the geodesic equation (8) to describe the moving of the shell [5]. However, there is a problem that in the nonstationary space-time the energy of a particle travelling on the geodesics is not conserved because of the space-time without a time-like killing vector field. So the energy of shell $\omega$ will vary when $v$ increases towards the future. The crucial point is that particle tunnelling a barrier is a instantaneous process. So we can fix a certain time $v^{\prime}$ and the energy of the black hole is $m\left(v^{\prime}\right)$ at the time $v^{\prime}$. When the positive energy virtual particle with energy $\omega$ just inside the event horizon tunnels just outside the event horizon where it materializes as a real particle, its energy is also $\omega$. At the same time the total energy of the black hole changes to $m\left(v^{\prime}\right)-\omega$ and the black hole shrinks a little. The line element (2) should be rewritten as

$$
\begin{aligned}
d s^{2}= & -\left\{1-8 \pi \eta^{2}-\frac{2\left[m\left(v^{\prime}\right)-\omega\right]}{r}-2 \dot{r}_{H}\right\} d v^{2} \\
& +2 d v d R+r^{2}\left(d \theta^{2}+\sin ^{2} \theta d \varphi^{2}\right) .
\end{aligned}
$$




\section{Tunnelling across the Horizon}

In our picture, a point particle description is appropriate. Because of the infinite blue shift near the horizon, the characteristic wavelength of any wave packet is always arbitrarily small there, so that the geometrical optics limit becomes an especially reliable approximation. The geometrical limit allows us to obtain rigorous results directly in the language of particles, rather than having to use the second-quantized Bogolubov method. When the black hole varies slowly, in the semiclassical limit, we can apply the WKB formula. This relates the tunnelling amplitude to the imaginary part of the particle action at stationary phase. The emission rate, $\Gamma$, is the square of the tunnelling amplitude $[4,18]$ :

$$
\Gamma \sim \exp (-2 \operatorname{Im} S)
$$

The imaginary part of the action for an outgoing positive energy particle which crosses the horizon outwards from $r_{\text {in }}$ to $r_{\text {out }}$ can be expressed as

$$
\operatorname{Im} S=\operatorname{Im} \int_{R_{\text {in }}}^{R_{\text {out }}} p_{R} d R=\operatorname{Im} \int_{R_{\text {in }}}^{R_{\text {out }}} \int_{0}^{p_{R}} d p_{R}^{\prime} d R,
$$

where $p_{R}$ is canonical momentum conjugate to $R$. In the coordinates $(v, R, \theta, \varphi), R_{i n}=$ $\left.r\right|_{r_{H}}-r_{H}=0$ is the initial radius of the black hole, and $R_{\text {out }}=\tilde{r}_{H}-r_{H}=-\delta$ is the final radius of the hole, where $\tilde{r}_{H}=r_{H}\left[m\left(v^{\prime}\right)-\omega\right]$. We substitute Hamilton' equation $\dot{R}=\left.\frac{d H}{d p_{R}}\right|_{R}$ into (11), change variable from momentum to energy, and switch the order of integration to obtain

$$
\begin{aligned}
\operatorname{Im} S & =\operatorname{Im} \int_{m}^{(m-\omega)} \int_{R_{\text {in }}}^{R_{\text {out }}} \frac{d R}{\dot{R}} d H \\
& =\operatorname{Im} \int_{0}^{\omega} \int_{R_{\text {in }}}^{R_{\text {out }}} \frac{2 d R}{1-8 \pi \eta^{2}-\frac{2\left[m\left(v^{\prime}\right)-\omega^{\prime}\right]}{r}-2 \widetilde{\dot{r}}_{H}}\left(-d \omega^{\prime}\right),
\end{aligned}
$$

where $\widetilde{\dot{r}}_{H}=\dot{r}_{H}\left(m\left(v^{\prime}\right)-\omega^{\prime}\right)$ We have used the modified equation (8) and $H$ is the Bondi energy of the space-time [4]. The radiative field do not radiate energy in the tunnelling process because a certain moment $v^{\prime}$ is fixed to study the tunnelling process, while the difference of the Bondi energy of the black hole of two different moments $v_{1}$ and $v_{2}$ is the energy radiated from the black hole during $v_{2}-v_{1}$. So we could get $d H=d\left[m\left(v^{\prime}\right)-\omega\right]=$ $-d \omega$ and the minus sign appears. Now let us explain the problem why we must adopt the new coordinates. If we do not adopt the new coordinates, (12) will be

$$
\operatorname{Im} S=\operatorname{Im} \int_{0}^{\omega} \int_{r_{\text {in }}}^{r_{\text {out }}} \frac{2 d r}{1-8 \pi \eta^{2}-\frac{2\left[m\left(v^{\prime}\right)-\omega^{\prime}\right]}{r}}\left(-d \omega^{\prime}\right) .
$$

The integral singularity(the first order pole) is at $r=2\left[m\left(v^{\prime}\right)-\omega^{\prime}\right] /\left(1-8 \pi \eta^{2}\right)$ where is time like limit surface, not event horizon. This means that the particle tunnels out of the time like limit surface, not of the event horizon. However, it is well known that Hawking radiation comes from the event horizon, not from the time like limit surface. In (12), $1-8 \pi \eta^{2}-\frac{2\left[m\left(v^{\prime}\right)-\omega^{\prime}\right]}{r}-2 \widetilde{\dot{r}}_{H}$ equals zero at event horizon. So we adopt the new coordinate and obtain (12) in order that the integral singularity is at $r=r_{H}^{\prime}$, where $r_{H}^{\prime}=r_{H}\left(m\left(v^{\prime}\right)-\omega^{\prime}\right)$. We integrate over $R$ firstly. The integral can be done by deforming the contour, so as to ensure that positive energy solutions decay in time (that is, into the lower half $\omega^{\prime}$ plane) [4]. In 
this way we obtain

$$
\operatorname{Im} S=\int_{0}^{\omega} \frac{4 \pi\left[m\left(v^{\prime}\right)-\omega^{\prime}\right] d \omega^{\prime}}{\left(1-8 \pi \eta^{2}-2 \widetilde{\dot{r}}_{H}\right)^{2}} .
$$

Because $\widetilde{\dot{r}}_{H}$ is the function of $\omega^{\prime}$ to the non-stationary black hole, it is difficult to work out the integral . However, we could make the physical meaning clear by the following method. The entropy of the black hole is $S_{B H}=\frac{4 \pi m^{2}\left(v^{\prime}\right)}{\left(1-8 \pi \eta^{2}-2 \dot{r}_{H}\right)^{2}}$ [12] and its derivative of $m\left(v^{\prime}\right)$ is

$$
\begin{aligned}
\frac{d S_{B H}}{d m\left(v^{\prime}\right)} & =\frac{8 \pi m\left(v^{\prime}\right)}{\left(a^{2}-2 \dot{r}_{H}\right)^{2}}+\frac{16\left[m\left(v^{\prime}\right)\right]^{2}}{\left(a^{2}-2 \dot{r}_{H}\right)^{3}} \frac{d \dot{r}_{H}}{d m\left(v^{\prime}\right)} \\
& =\frac{8 \pi m\left(v^{\prime}\right)}{\left(a^{2}-2 \dot{r}_{H}\right)^{2}}-2 S_{B H} \frac{d}{d m\left(v^{\prime}\right)} \ln \left(a^{2}-2 \dot{r}_{H}\right),
\end{aligned}
$$

where $a^{2}=1-8 \pi \eta^{2}$. Doing the integral of $m\left(\tilde{v}^{\prime}\right)$, (15) could be written as

$$
\begin{aligned}
& \int_{m\left(v^{\prime}\right)}^{m\left(v^{\prime}\right)-\omega} \frac{8 \pi m\left(\tilde{v}^{\prime}\right)}{\left(a^{2}-2 \dot{r}_{H}\right)^{2}} d m\left(\tilde{v}^{\prime}\right) \\
& \quad=\int_{m\left(v^{\prime}\right)}^{m\left(v^{\prime}\right)-\omega} \frac{d S_{B H}}{d m\left(\tilde{v}^{\prime}\right)} d m\left(\tilde{v}^{\prime}\right) \\
& \quad+\int_{m\left(v^{\prime}\right)}^{m\left(v^{\prime}\right)-\omega} 2 S_{B H} \frac{d}{d m\left(\tilde{v}^{\prime}\right)} \ln \left(a^{2}-2 \dot{r}_{H}\right) d m\left(\tilde{v}^{\prime}\right) .
\end{aligned}
$$

It is obvious that the term of the left-hand side of (16) is just $-2 \operatorname{Im} S$ in (14) and the first term of the right-hand side of (16) is just $\Delta S_{B H}=S_{B H}\left[m\left(v^{\prime}\right)-\omega\right]-S_{B H}\left[m\left(v^{\prime}\right)\right]$. So, the term of the left side of (16) corresponds to the exponent of $\Gamma$ and on the right-hand side of (16) there is a remainder $\int_{m\left(v^{\prime}\right)}^{m\left(v^{\prime}\right)-\omega}\left[-2 S_{B H} \frac{d}{d m\left(v^{\prime}\right)} \ln \left(1-8 \pi \eta^{2}-2 \dot{r}_{H}\right) d m\left(\tilde{v}^{\prime}\right)\right]$ except $\Delta S_{B H}$, which is different from Parikh's result.

\section{Conclusion and Discussion}

The tunnelling rate obtained in this paper is the function of time $v^{\prime}$ which describes the black hole itself. Moreover, when $m$ is a constant and $\eta=0$, it could come back to the result of the static Schwarzschild black hole. So, our results reflect the dynamical change of the non-static black hole with the internal global monopole. In our opinion, this result is reasonable and anticipatory. When we study the tunneling effect from a black hole, there exists thermal equilibrium between the black hole and its environment in the case of the static and stationary space-time. So, Parikh get the result, $\Gamma \sim \exp \left(\Delta S_{B H}\right)$ [5]. However, because of the radiation of the black holes the non-stationary black holes exist more generally. When black hole is non-stationary, it is impossible that the black hole keeps thermal equilibrium between the black hole and its environment. In addition, an important difference between stationary and non-stationary black holes is whether $r_{H}$ is the function of time. Due to nonstationary black holes, the event horizon $r_{H}$ and the entropy of the black hole are related not only to the Bondi energy but also to $\dot{r}_{H}$. Consequently, the variation of $r_{H}$ and the entropy also depends on $\dot{r}_{H}$ when $m\left(v^{\prime}\right)$ changes.

Acknowledgement This work is supported by the National Natural Science Foundation of China under Grand No.10773002 and the National Basic Research Program of China (No: 2003CB716300). 
Open Access This article is distributed under the terms of the Creative Commons Attribution Noncommercial License which permits any noncommercial use, distribution, and reproduction in any medium, provided the original author(s) and source are credited.

\section{References}

1. Hawking, S.W.: Common. Math. Phys. 43, 199 (1975)

2. Kraus, P., Wilczek, F.: Nucl. Phys. B 433, 403 (1995)

3. Parikh, M.K.: A secret tunnel through the horizon. hep-th/0405160 (2004)

4. Parikh, M.K.: Energy conservation and Hawking radiation. hep-th/0402166 (2004)

5. Parikh, M.K., Wilczek, F.: Phys. Rev. Lett. 85, 5042 (2000)

6. Hemming, S., Keski-Vakkuri, E.: Phys. Rev. D 64, 044006 (2001)

7. Medved, A.J.M.: Phys. Rev. D 66, 124009 (2002)

8. Jingyi, Z., Zheng, Z.: Hawking radiation of charged particle via tunneling from the Reissner-Nordstrom black hole. J. High Energy Phys. 10, 055 (2005)

9. Zhang, J., Zhao, Z.: Massive particles black hole tunneling and de sitter tunneling. Nucl. Phys. B 725, 173-180 (2005)

10. Zhang, J., Zhao, Z.: Charged particles' tunneling form the Kerr-Newman black hole. Phys. Lett. B 638, 110-113(2006)

11. Zhang, J., Fan, J.-H.: Tunnelling Effect of Charged and Magnetized Particles from the Kerr-NewmanKasuya black hole. Phys. Lett. B 648, 133-138 (2007)

12. Yu, H.: Phys. Lett. A 182, 353-355 (1993)

13. Han, Y., Liu, S.: Chin. Phys. Lett. 22(7), 1608-1610 (2005)

14. Zhang, H., Zhao, Z.: J. Beijing Norm. Univ. (Nat. Sci.) 37, 471 (2001) (in Chinese)

15. Zhang, J.Y., Fan, J.H.: Physica A 379, 90-100 (2007)

16. Zhang, J.Y., Fan, J.H.: Phys. Lett. B 648, 133-138 (2007)

17. Zhang, J.Y., Fan, J.H.: Mod. Phys. Lett. A 22, 1821-1828 (2007)

18. Zhang, J.Y., Fan, J.H.: Chin. Phys. 16, 3879-3884 (2007) 